\title{
LEXICO Y CONFIGURACIÓN SEMÁNTICA EN LATÍN: LA NOCIÓN DE 'EXTENSIONALIDAD'
}

Leonor Molero Alcaraz

1.1. Si se aborda el léxico de cualquier lengua como materia que puede ser objeto de estudio científico y riguroso, de forma similar a como se investiga en otros planos lingüísticos, la primera dificultad de peso radica obviamente en el inmenso número de unidades analizables. Ello conlleva el que mientras las restantes parcelas - la fonéticofonológica, la morfológica y la sintáctica, donde las unidades estudiadas son incomparablemente menos numerosas -, se conocen y se han descrito a lo largo de toda una tradición linguiística dentro de cada lengua, el estudio del léxico está, por su propia naturaleza, condicionado a llevarse a cabo siempre de forma fragmentaria, trabajándose en pequeñas zonas que sólo dejan entrever un minúsculo aspecto, en cada una, de lo que cabe relacionar con la totalidad. Ello es lo que se ha logrado hasta ahora mediante la labor de diversos investigadores del léxico latino ${ }^{1}$, cuyas valiosas aportaciones han brindado la posibilidad de acceder a determinadas figuras de un mosaico inconmensurable, el léxico latino, disperso en los textos y compilado en las obras lexicográficas; en éstas, el léxico conservado de la lengua latina aparece más veces en forma de catálogo o repertorio exhaustivo que como corpus organizado, donde los elementos forman parte de un todo, en el que a menudo se relacionan directamente entre sí. Ahora bien, no tenemos más que la visión de conjunto aportada por los grandes diccionarios para intentar el conocimiento global del léxico de una lengua, y tal debería ser el punto de partida para cualquier tipo de trabajo sobre el léxico, y así proceder al análisis en la realización discursiva - los textos en el caso de una lengua como el latín -.

1.2. El estudio del léxico en la línea de la lingüística estructural, propugnado por E. Coseriu o B. Pottier, aun con sus diferencias de criterios y de postulados teóricos, ha conseguido abrir en los últimos veinte años una brecha por donde emprender una investigación coherente del material léxico, partiendo de lo que realmente éste representa en la lengua: no una inabarcable masa de elementos que se acumulan unos tras otros, sino un 
sistema donde los elementos se jerarquizan y, sobre todo, tienen una funcionalidad determinada, dentro de una totalidad compleja.

A este respecto, resulta útil la distinción que establece E. Coseriu ${ }^{2}$ entre la palabra lexemática, como portadora de la función léxica, y otros tipos de términos registrados en los dicccionarios: nombres terminológicos, palabras gramaticales, etc. Tal distinción sitúa al investigador del léxico en el objeto inmediato de su estudio, representado por las palabras que participan de lo lingüísticamente funcional; al mismo tiempo, como no todas las palabras del léxico son lexemáticas en el sentido establecido por dicho autor, se reduce en alguna medida el número de elementos analizables.

Basándose en otra matización decisiva, que se refiere a la diferencia entre significado y designación ${ }^{3}$, para E. Coseriu términos lexemáticos son aquellos que implican un grado específico de abstracción nocional, las palabras que cada lengua crea confiriéndoles un contenido determinado; mediante este tipo de elementos léxicos, una lengua organiza su genuina respuesta a cuanto existe fuera de ella, a través de los distintos significados léxicos. En el caso de los lexemas o palabras lexemáticas, la lengua no propone la simple denominación de las cosas, como mera representación oral de las mismas - una especie de sustituto fónico -, que es lo que sucede con los nombres propios o con las terminologías y nomenclaturas; por el contrario, en las palabras lexemáticas se configura a menudo una visión o interpretación singular del referente, al que se presenta de manera peculiar en una lengua concreta: de este modo, cuando en el latín de la lengua poética se designa la tierra, y ésta es entendida como fuerza viva y productora de cuanto existe sobre ella, se dispone del significado de tellus.

Una cantera para la investigación de palabras lexemáticas se ofrece con frecuencia en el fenómeno de la sinonimia, donde por economía lingüística habría que descartar los sinónimos completos y totales, al menos en un estadio inicial de creación de palabras: todos los sinónimos aportarían un rasgo significativo, que justificara la propia existencia de la palabra.

Sin embargo, el marco preferido por la semántica estructural para el estudio del léxico es, como se sabe, el campo semántico, una estructura en la que sólo entra un reducido número de elementos con función léxica y que participan de una base de significado común. Fuera quedan palabras que funcionan como simples nombres de las cosas -nombres de árboles, de objetos, de animales, etc.; verbos y adjetivos de significado restringido a lenguas técnicas, como la de la agricultura o el derecho, etc. -; tales términos, si bien hay que reconocerles la sola función de nomenclaturas, no enriquecen menos el léxico, en su diversidad y exuberancia formal, que las llamadas palabras lexemáticas.

\footnotetext{
2 E. Coseriu, Principios de Semántica Estructural, vers. M.Martínez Hernández, rev. por el autor, Madrid, 1977; 2 ed. 1986 (1981), pp. 88 ss.

${ }^{3}$ E. Coseriu, op.c., capítulo V ("Significado y designación a la luz de la Semántica Estructural"), pp. 185 ss.
} 
1.3. Cuanto va a exponerse en este trabajo ${ }^{4}$ se refiere a términos lexemáticos del léxico latino, o, mejor dicho, a un rasgo sémico que los define semánticamente, organizando una serie de relaciones opositivas; dichos términos se agrupan en lo que puede denominarse "campo semántico 'tierra'", especialmente analizado en las Geórgicas de Virgilio. Pretendo con ello añadir una demostración más de que también este plano de la lengua es susceptible de análisis lingüístico, y de que las palabras no existen en el léxico amalgamadas sin más unas junto a otras, sino con alguna articulación orgánica que es preciso descubrir.

2.1. Por la noción o rasgo sémico que convencionalmente denomino "extensionalidad" o "extensional", los significados de varios términos del campo semántico "tierra" presentan el ente extralingüístico tierra como "superficie cerrada o delimitada en sí misma", es decir, como "extensión"; por el contrario, otro grupo de elementos léxicos no marcan dicha noción de "tierra como superficie extensional con un área propia", y sin embargo sí expresan un rasgo diferente: el de "la tierra como superficie que sostiene, sobre la que se sitúan los seres y las cosas materiales". Se trata, pues, del dualismo nocional "tierra-extensión" / "tierrasuelo"; del primer rasgo participan diversos términos que pueden clasificarse como "extensionales" - campus, aruum, ager, litus, rus, ripa, aequor, seges, area, deserta, noualis, planum, por orden de frecuencia decreciente en las Geórgicas virgilianas -. Por una especie de polaridad, cabe hablar de términos "no-extensionales", que son terra, tellus, humus, solum, harena, limus, puluis, creta y tofus, enumerados también de más a menos contextos en que aparecen dentro del mismo poema. Como esta segunda serie comparte el rasgo "tierra-suelo", frente a los de la primera, definidos por la noción "tierra-extensión", el tipo de relación más probable entre los elementos de uno y otro grupo revestiría la forma de la oposición equipolente ${ }^{5}$, algo que comprobaremos analizando los textos.

2.2. La noción sémica "extensionalidad" parece sugerida no sólo por la observación directa del comportamiento léxico de los términos, en los textos; igualmente, se conservan diversas referencias en autores antiguos que, a modo de valiosísima documentación lexicológica, parece que sustentan la formulación de lo "extensional" como rasgo semántico.

Con respecto al menos marcado de los "extensionales", ager, resultan interesantes las siguientes definiciones técnicas: strigatus ager est, qui a septentrione in longitudinem in meridianum decurrit; scamnatus autem, qui eo modo ab occidente in orientem crescit (Hyg.

${ }^{4}$ El tema desarrollado se basa en una comunicación que, con el título "La noción sémica 'extensional' en los términos virgilianos del campo semántico 'tierra'", fue presentada al II Congreso Andaluz de Estudios Clásicos, celebrado en Antequera- Málaga en 1984; las Actas de dicho Congreso no se han terminado de publicar hasta 1994, apareciendo el trabajo en cuestión en el vol. III (pp. 103-109), y sin haber tenido corrección de pruebas de imprenta.

5 Supone ello un cambio con respecto a un análisis anterior, según se recogía en la comunicación presentada al II Congreso Andaluz de EE.CC.; allí este tipo de oposición entre un término "no-extensional" y otro "extensional" - por ejemplo, terra(-)/ager(+) -, se proponía como oposición privativa, lo que no quedaba refrendado por el comportamiento lexicológico de los términos en los textos virgilianos. 
Grom. ed. Lachmann, p.110); arcifinius ager dictus est qui a certis linearum mensuris non continetur, sed arcentur fines eius obiectu fluminum, montium, arborum...(Isid.Orig.15,13,11). En ambos lugares se utilizan, a nivel sintagmático, expresiones que apuntan a la idea de "extensión" implícita en ager: decurrit, crescit; a certis... mensuris NON CONTINETUR. No menos explícita resulta la definición lexicográfica de Forcellini, s.v. ager: ager proprie est terra culta manibus hominum, ut fruges ferat, sive hoc generatim sumatur sive speciatim DE PORTIONE TERRAE DEFINITA quae coli possit.

En cuanto al "extensional" más frecuente en las Geórgicas, campus, también nos aporta algo de luz la siguiente aclaración de Verecundo (In Cant. 6,6): campus planities est terrae latitudine longiore DISTENTA, in quibus nullus collis nec mons nec locus altior invenitur; sed distenta tantum aequalitas, ampliori latitudine ultro citroque DIFFUSA. Mucho más clara la precisión isidoriana: campus est terrarum planities, dictus autem campus, quod sit brevis pedibus nec erectus, ut montes, sed PATENS et SPATIO SUO PORRECTUS et IACENS...(Isid. Orig. 14,8,23). Ya en un ejemplo de Ovidio se recoge la idea de "extensionalidad" inherente a un término como campus: (deus) iussit et EXTENDI campos, subsidere ualles... / surgere montes (Ov. Met.1,43). La vinculación semántica entre el verbo extendere y el significado de campus, si se admite como rasgo distintivo de dicho significado la noción de "extensional" - vinculación que por otra parte también se produce entre subsidere-ualles, surgere-montes -, relaciona esta serie de elementos con lo que Coseriu denomina estructura sintagmática de "implicación" 6: el lexema verbal funciona como rasgo distintivo del sustantivo.

Una tercera referencia en la que sustentamos la noción analizada nos la proporciona el Thesaurus Linguae Latinae para el término litus (s.v.): designatur TRACTUS harenosus molliter devexus...; respicitur litoris pars sicca: strictius respicitur AREA harenosa, quae mare terminat.

2.3. Una misma designación, entendida como ente que existe fuera de la lengua, puede expresarse mediante significados lingüísticos diferenciados, los cuales se apoyan en distintos significantes que en principio no tienen por qué corresponder a sinónimos banales, en una especie de derroche léxico; al menos en su origen, los términos de significado próximo se diferencian entre sí por algún matiz específico, aunque luego dejen de hacerlo como consecuencia de interferencias semánticas que muy bien podemos identificar con los llamados usos neutros. Como veremos un poco más adelante, en el texto virgiliano sobre el que ejemplificamos este análisis, a la tierra de cultivo se le puede llamar terra o humus y, en otra ocasión, ager, arua o campus; para una playa, encontramos en latín unas veces terra o harena, y, el término propio, litus.

\footnotetext{
${ }^{6}$ E. Coseriu, op. c., pp. 141, 183; E. Coseriu, Gramática, Semántica, Universales (Estudios de Lingüística Funcional), trad. M.Martínez Hernández, rev. por el autor, Madrid, 1978, pp. 232-233.
} 
Que la tierra-campo (espacio para la agricultura) unas veces se decía terra y otras ager, se muestra en el siguiente comentario de Servio: nam omnis TERRA, ut etiam Varro docet, quadrifariam dividitur: aut enim arvus est AGER, id est, sationalis; aut consitus, id est, aptus arboribus; aut pascuus, qui herbis tantum et animalibus vacat; aut floreus, in quo sunt horti apibus congruentes et floribus ${ }^{7}$.

Pero es que aun sin salir de un mismo contexto virgiliano se da este mismo fenómeno de diversidad de significados para una misma designación: difficiles primum TERRAE COLLESque maligni / tenuis ubi argilla et dumosis calculus ARVIS / Palladia gaudent silua uinacis oliuae: / indicio est tractu surgens oleaster eodem / plurimus et strati bacis siluestribus AGRI (Georg. 2, 179-183). De paso, los términos tractus y sternere inciden sobre la idea de lo "extensional".

Ante tal coincidencia designativa, no es de extrañar que ager deba traducirse a veces por "tierras" - que en español también incluiría la noción "extensional" cuando se construye en plural -: militibus...AGROS ex suis possessionibus pollicetur (Caes. Civ.1,17,4), "a sus soldados... les promete TIERRAS (cedidas) de sus propias posesiones" ${ }^{8}$. En las Geórgicas encontramos un ejemplo similar al anterior: ... si pinguis AGROS metabere campi / densa sere (2, 274-275), "si acotas las TIERRAS de una llanura rica, siembra junto...". En este contexto, los términos ager y campus no resultan redundantes porque cada uno se polariza en su valor propio de lengua: ager, "tierra-extensión-delimitada" frente a campus, "tierraextensión-llana (sin desniveles)", correspondiendo en, variatio léxica, al término ocasional planum, que aparece en el verso 2,273.

También en Georg. 2,214-216: et tofus scaber et nigris exesa chelydris / creta negant alios aeque serpentibus AGROS / dulcem ferre cibum et curvas praebere latebras. En este lugar, el "extensional" ager ocupa, frente a los "no-extensionales" tofus y creta, una posición funcional claramente conmutable con terra, si bien no necesariamente en uso neutro: por polarización, ager puede seguir significando "extensión", mientras los dos primeros significan "suelo" o, mejor, "tipos de suelo". Tal situación léxica convendría reflejarla en la traducción: "la toba escabrosa y la greda minada de negras culebras: dicen que ningunas otras TIERRAS producen igual apetecible sustento ni igual proporcionan sinuosos escondrijos a las serpientes".

2.4. La relación entre el "extensional" ager y el "no-extensional" terra se produce como oposición equipolente, que parece el tipo más abundante entre las oposiciones léxicas; ambos términos se contraponen bajo la doble noción de "tierra-extensión" ager(+) / "tierrasuelo" terra $(+)$.

7 Thilo, G. - Hagen, H. (edd.), Servii grammatici qui feruntur in Vergilii Bucolica et Georgica Commentarii, III 1, Hildesheim, 1961 (=Leipzig, 1887), pp. 128-129.

8 G. Julio César, Memorias de la Guerra Civil, tex. rev. y trad. por S. Mariner Bigorra, Barcelona, 1959 y 1961, vol. I, pp. 20-21. 
En las Geórgicas se dan varios contextos similares donde cada uno de estos dos términos actualizan sus valores propios: para ager, 1,$102 ; 1,107 ; 2,6 ; 2,236 ; 2,330 ; 1,81 ; 1,84$; 1,$123 ; 2,54 ; 2,346$. Para terra, 2,203; 2,287; 1,$1 ; 1,119 ; 1,147 ; 1,494 ; 2,237 ; 2,259$; 2,$513 ; 3,160 ; 3,534 ; 1,63 ; 1,83 ; 1,224 ; 2,29 ; 2,27 ; 2,313 ; 1,86 ; 1,184 ; 1,479 ; 2,37$; 2,$109 ; 2,179 ; 2,324 ; 3,429 ; 3,432 ; 3,525 ; 2,92$.

Lo mismo cabe decir si se oponen campus (+) y terra (+), bajo la misma noción; en algunos de estos ejemplos, resulta significativo el que aparezcan en combinatoria sintagmática con los mismos verbos, cuando uno y otro término actualizan el rasgo "para la agricultura": uertere e inuertere se aplican por igual a terra $(1,1 ; 1,63 ; 3,525)$ y a campus $(3,161)$. En estos últimos ejemplos, la diferencia de significado estriba en presentar la tierra como suelo o como superficie delimitada que, en cualquier caso, se labra: quid uomere TERRAS inuertisse grauis? $(3,525)$ / (...quos... malint...)/... scindere terram et CAMPUM horrentem fractis inuertere glaebis (3, 160-161).

Con el verbo mouere, o su compuesto dimouere, para la misma idea de cultivar la tierra, se produce idéntica diferencia que en el caso anterior, cuando el par de términos considerados son terra y ager: pater...primus...per artem mouit AGROS $(1,123)$ / agricola incuruo TERRAM dimouit aratro $(2,513)$.

2.5. Las oposiciones equipolentes que se articulan en torno a la noción de "extensionalidad", entre términos "extensionales" y "no-extensionales", se repiten en varias series de términos: terra y litus, harena y litus, harena y deserta, puluis y deserta. Algunos ejemplos: nudus in ignota, Palinure, iacebis HARENA (Aen. 5,871) // ...uotaque seruati soluent in LITORE nautae (Georg. 1,436); fluctus...ut... uolutus ad TERRAS immane sonat per saxa (Georg. 3, 239) I/ ...longi urgent ad LITORA fluctus (Georg. 3,200); totaque turiferis Panchaia pinguis HARENIS (Georg.2,139) // pascitur itque pecus longa in DESERTA sine ullis / hospitiis: tantum campi iacet. (Georg. 3, 342-343). Este último contexto también se contrapone en cierta medida a 4,96: ...ceu PULVERE ab alto quom uenit et sicco terram spuit ore uiator aridus, "(las abejas obreras de feo aspecto) como la TIERRA que escupe un caminante sudoroso cuando llega de una profunda POLVAREDA", lo que bien puede referirse a quien acaba de atravesar una extensión de tierra reseca, como el desierto, aunque caben otras designaciones posibles.

2.6. Los usos de terra y ager, tomados como términos representativos de sus series respectivas, son normalmente los propios de estos dos lexemas; en singular, terra se diferencia de ager por su significado propio, e incluso en plural, terra no pasa a significar "tierra-extensión" - lo que sugeríamos que sí ocurre en español -. La relación de equivalencia lógica es tan neta entre ambos términos que cuando estos sustantivos se construyen en plural, se distribuyen matices significativos especiales: terra en plural contiene a menudo el rasgo "suelo terrestre (por oposición al resto del Universo, mundus)", como se ve en una 
serie de contextos de las Geórgicas, a los que remitimos: 1,288; 2,345; 4,52; 4,222; 2,479; 4,$232 ; 2,474 ; 2,538 ; 3,242$. A su vez ager, por su propio contenido de "extensión delimitada" se especializa a menudo para el valor de la tierra como algo que constituye la posesión de un individuo o una comunidad, equivaliendo no ya a "terreno" o "campo", sino a "territorio, demarcación territorial"; en este caso aparece tanto en singular como en plural: en las Geórgicas, 1,471; 4,522 y 3,249. Cuando el territorio no se refiere a una comarca, sino a una extensión mayor - la ocupada por un país -, se prefiere terra, en singular y ya claramente en uso neutro; resulta ilustrativa al respecto la siguiente aclaración de Varrón: ut ager Tusculanus, sic Calydonius ager est, non terra (Ling. 7,18). Del mismo uso neutro de terra por ager cuando se designa un país encontramos un ejemplo en 2,136: ... Medorum siluae ditissima terra.

En 3,171 creo que es preferible considerar un uso neutro de terra por ager, bastante condicionado por el valor de la preposición per, cuyo significado es "a través/ por medio de" (lo que implica que regirá un contenido que incluya la idea de "extensión"): atque illis (uitulis) iam saepe rotae ducantur inanes / per TERRAM, et summo uestigia puluere signent. En este caso, si lo interpretamos como uso neutro, habría que traducir terra por "campo": "y que con su esfuerzo (de los becerros) se tire a menudo de ruedas ligeras (carros vacíos) por medio del CAMPO, y apenas dejen señaladas sus huellas en lo alto del suelo polvoriento".

A la inversa, también es posible el uso neutro de ager por terra, y de ello encontramos un curioso ejemplo en las Geórgicas (2, 239-244): salsa autem tellus et quae perhibetur amara / (...) / (...) / tale dabit specimen. tu spisso uimine qualos / colaque prelorum fumosis deripe tectis; / huc AGER ille malus dulcesque a fontibus undae ad plenum calcentur... "a su vez la tierra salina, y la que se conoce como amarga, te dará la siguiente prueba. Baja tú de los techos ahumados cestas de espeso mimbre y coladores de los lagares; que se apisonen aquí la TIERRA de aquel SUELO malo y agua dulce de la fuente...". Ante un caso como éste, ya los comentaristas de los Scholia Bernensia llamaron al sentido lógico: ager, terra ${ }^{9}$; y es que sin recurrir a una figura metonímica de contenido por continente, difícilmente se puede meter un campo en un cesto, para que deje su sabor en el agua con que se mezcla.

Sin embargo, tal uso retórico está permitido por lo que considero un uso neutro de ager, que esta vez actualiza el valor de terra, porque no se incide tanto en la idea de "extensión o terreno" como en la de "superficie o suelo", sobre todo, "tipo de suelo": tellus salsa et amara. Un segundo paso se produce cuando ager - funcionando con el valor de terra o humus -cambia de "dimensión semántica" 10: de "tierra-superficie" (la dimensión central de nuestro

${ }^{9}$ H. Hagen (ed.), Scholia Bernensia ad Vergili Bucolica atque Georgica, Hildesheim, 1967 (=Leipzig, 1867), p. 235.

10 Para el concepto de dimensión semántica, véase H. Geckeler, Semántica estructural y teoría del campo léxico, vers. M.Martínez Hernández, Madrid, 1976, r. 1984, pp. 298-299. 
campo), pasa a "tierra-masa", por lo que funciona como lexema ocasional dentro de esta otra zona del campo léxico estudiado.

3. Se observa, a partir de los datos anteriores, que términos comunes, como los analizados en el ámbito léxico de lo que puede considerarse un campo semántico, no se limitan a aparecer como posibles recursos del hablante latino a la hora de referirse a la tierra que habitaban, cultivaban y poseían; cada uno aporta además su propio contenido, justificándose con ello su existencia en la lengua. Unicamente como consecuencia del uso frecuente pueden llegar a interferirse y a desviarse de su valor original -al igual que se modifican los sonidos o los sistemas morfosintácticos, a lo largo del tiempo- propiciando lo que a veces desemboca en un cambio de significado.

En las Geórgicas virgilianas, terra es el lexema más frecuente, y funciona con dos valores básicos, en dos zonas léxicas (dimensiones) que corresponden a las nociones de "tierra-superficie" y "tierra-masa". No obstante, son mucho más numerosos sus usos propios que los usos neutros por otros términos.

De la misma manera, el buen poeta latino recurre a la variatio léxica, mas sin caer en la sinonimia banal o relleno métrico; cuando en un mismo contexto aparecen dos o más términos del campo semántico "tierra", cada uno se polariza en su uso propio, como muestra este ejemplo de Tibulo: totosque per agros floret odoratis terra benigna rosis $(1,3,61)$, "y por todos los vergeles florece la tierra bienhechora con sus rosas perfumadas" si recordamos la definición de Servio, una clase de ager ("terreno") era el floreus, que corresponde a lo que en español es un jardín, huerto o vergel, con la base semántica de "tierra-extensión" -; a su vez, terra aúna aquí a su significado "tierra-suelo" un rasgo de "animación", facilitado por la combinatoria sintagmática con el verbo floreo y con el adjetivo benignus.

Resumiendo mi exposición, la noción de "extensionalidad" constituye un rasgo destacado que define la configuración común del significado de una serie de términos, referidos a la tierra; pese a ello, es evidente que si se busca la completa definición semántica de cada elemento hay que contar con otros rasgos más. Por el momento, sirva de muestra el análisis textual de dicha noción, para una alternativa a la manera en que tradicionalmente se ha estudiado el léxico latino. 